Orange Journal/Volumen 1 Número 1/ Enero - Junio 2019

DOI: http://dx.doi.org/issn.2710-995X/2019.1.02

\title{
Los proyectos metodológicos como método para el aprendizaje de futuros maestros
}

\author{
Projetos metodológicos como método para a aprendizagem de futuros professores \\ Recibido: 05 de febrero de 2019 \\ Aceptado: 29 de marzo de 2019 \\ Escrito por: \\ Zimfir Z. Rizvanov ${ }^{3}$ \\ Vera S. Abaturova ${ }^{4}$ \\ Irina E. Malo ${ }^{5}$ \\ Liliana R. Shakirova ${ }^{6}$
}

\section{Resumen}

La búsqueda de los medios efectivos para mejorar la formación de un profesor de matemáticas condujo a la idea de crear proyectos por parte de los estudiantes demostrando la metodología del trabajo del profesor con el alumnoenla tarea, el teorema o el textodel librode texto. El artículo resume los resultados del estudio experimental.

Los métodos de experiencia subjetiva colectiva y modelado visual se consideraron básicos. El formulario de presentación del proyecto fue una presentación en computadora, cuyos efectos de animación permiten la creación consistente de imágenes gráficas, así como la organización de una pausa en el diálogo del docente con los estudiantes.

Las condiciones para asegurar el éxito de los estudiantes en el desarrollo del proyecto son las siguientes:

1) Observancia de las etapas del método de experiencia colectiva del sujeto;

2) Formulación de la tarea al proyecto metódico, que refleje los requisitos para los resultados de su creación;

3) Razonabilidad de las decisiones metódicas según los requisitos del enfoque de la actividad y la capacitación orientada personalmente.

Los requisitos del enfoque de actividad son los siguientes:

1. Laexistenciadel objetivo como un resultado de actividad planificado;

2. Uso de bases indicativas de actividad, formuladas en forma generalizada;

3. Desarrollo de pasos individuales de actividad.

Los requisitos de la capacitación orientada a la persona son los siguientes:

1) Orientación de capacitación para enriquecer la experiencia de los estudiantes en el tema (Yakimanskaya, 2014).

2) Garantizar la posición de liderazgo de los estudiantes en la actividad cognitiva y reflexiva;

3) Cumplimiento de los requisitos para la organización del diálogo de formación

\footnotetext{
3 Kazan Federal University.

${ }^{4}$ Southern Mathematical Institute of the Vladikavkaz Scientific Center of the Russian Academy of Sciences.

${ }^{5}$ Bryansk State Academician I.G. Petrovski University.

${ }^{6}$ Kazan Federal University.
} 
(motivación de las preguntas, su naturaleza general, conectividad, enfoque y naturaleza del diálogo etapa por etapa, registro de la experienciapersonaldelosestudiantes, iniciativa de los estudiantes) y posibilidades del polílogo).

Palabras claves: Entrenamiento, matemática, tarea, teorema, texto, visibilidad, modelado, diálogo, proyecto.

\section{Resumo}

A busca por meios eficazes para melhorar a formação de um professor de matemática levou à ideia de criar projetos pelos alunos demonstrando a metodologia do trabalho do professor com o aluno no dever de casa, o teorema ou o texto do livro didático... O artigo resume os resultados do estudo experimental.

Os métodos de experiência subjetiva coletiva e modelagem visual foram considerados básicos. O formulário de apresentação do projeto foi uma apresentação de computador, cujos efeitos de animação permitem a criação consistente de imagens gráficas, bem como a organização de uma pausa no diálogo do professor com os alunos.

As condições para garantir o sucesso dos alunos no desenvolvimento do projeto são as seguintes:

1) Observância das etapas do método de experiência coletiva do sujeito;

2) Formulação da tarefa ao projeto metódico, que reflete os requisitos para os resultados de sua criação;

3) Racionalidade das decisões metódicas de acordo com os requisitos da abordagem da atividade e treinamento orientado para o pessoal.

Os requisitos de abordagem de atividade são os seguintes:

1) A existência do objetivo como resultado da atividade planejada;

2) Uso de bases indicativas de atividade, formuladas de forma generalizada;

3) Desenvolvimento de etapas de atividades individuais.

Os requisitos da formação orientada para as pessoas são os seguintes:

1) Orientação de treinamento para enriquecer a experiência dos alunos no assunto (Yakimanskaya, 2014).

2) Garantir a posição de liderança dos estudantes na atividade cognitiva e reflexiva;

3) Cumprimento dos requisitos para a organização do diálogo de formação (motivação das questões, natureza geral, conectividade, foco e natureza do diálogo, etapa por etapa, registo da experiência pessoal dos alunos, iniciativa do aluno) e possibilidades do polylogue).

Palavras-chave: Treinamento, matemática, lição de casa, teorema, texto, visibilidade, modelagem, diálogo, projeto

\section{Introducción}

El problema de la calidad de la capacitación metódica del futuro maestro siempre será de actualidad, ya que el cambio de cualquier condición de capacitación requiere encontrar nuevas formas de resolverlo. Uno de los medios para aumentar la efectividad de la capacitación metódica es el desarrollo de un proyecto metódico por parte del estudiante que cumpla con los requisitos modernos para el aprendizaje de los estudiantes: 
implementación del enfoque de actividad del sistema en la enseñanza de los estudiantes; Enseñanza de los alumnos orientada personalmente por la asignatura académica; utilizando la mediación de las tecnologías de la información y la comunicación (TIC) en la formación.

Se eligió una presentación por computador como medio para demostrar el modelado visual de una solución metódica (Smirnova y Yaroslavl, 2010). El proceso de su desarrollo basado en los requisitos de los métodos de enseñanza de los estudiantes para resolver los problemas y estudiar los teoremas en conjunto con el trabajo creativo individual y en la correlación de sus decisiones metódicas con su influencia en las consecuencias de la capacitación, permite llegar a una conclusión.

Que los proyectos metódicos pueden considerarse como un método de formación de futuros docentes.

El artículo (Holmqvist) corrobora el resultado del estudio, en el cual los maestros mejoraron a sí mismos cuando desarrollaron las lecciones de estudio de aprendizaje (Holmqvist, 2011). Un efecto similar se observa cuando los futuros maestros desarrollan los proyectos metódicos de enseñanza.

El artículo (Goos) demuestra el uso de la teoría histórico-cultural por parte de L.S. Vygotsky sobre el ejemplo de la enseñanza de las matemáticas en Australia (Goos, 2004). Identificamos los fundamentos de la reforma educativa, desde las prácticas de instrucción hasta los resultados del aprendizaje mediante la inclusión de los estudiantes en la comunicación, la búsqueda de formas de razonamiento y el poder de resolución de problemas. Este enfoque corresponde a las etapas de trabajo en tareas matemáticas en los proyectos metódicos y la inclusión de los estudiantes en el diálogo educativo.

El documento considera los diversos tipos de conocimiento: saber-eso, saber-cómo, saber-por qué, saber-sobre justifica que el saber-actuar en el momento pasa por todos estos tipos de conocimiento (Mason y Spence, 1999). El análisis de la relación entre el conocimiento de la asignatura del profesor y los logros de los estudiantes se dedica al estudio de Choshanov et al (Tchoshanov et al, 2017). Llama la atención sobre la necesidad de actualizar la propia experiencia como incentivo para la acción. En el método ruso de enseñanza de las matemáticas, este motivador para la acción se llama motivación. En los proyectos metódicos de los estudiantes, el requisito de motivación se aplica no solo a las acciones de los estudiantes, sino también a las acciones de un maestro, por ejemplo, cualquier pregunta de diálogo debe estar motivada, es decir, los estudiantes deben entender por qué esta pregunta se realiza; En la etapa de resumir los resultados de la actividad matemática, deben discutirse todos los métodos que motivan una acción / razonamiento particular. Cuando se utilizan proyectos metódicos en la formación de futuros docentes. 


\section{ORANGE JOURNAL}

\section{Métodos}

Método de experiencia de sujeto colectivo. Los métodos de experiencia subjetiva colectiva desarrollados por nosotros incluyen ocho etapas, su observancia asegura el éxito del proyecto:

1. Se actualiza la experiencia de los alumnos al trabajar con un objeto matemático (tarea, teorema, texto de un libro de texto).

2. El método de modelado visual estudia los problemas de la metodología general de trabajo con un objeto matemático: etapas de trabajo, implementación del enfoque de la actividad, aprendizaje orientado personalmente.

3. En el trabajo colectivo, la teoría se aplica al análisis de desarrollos / muestras de fragmentos específicos de la lección bajo la guía del maestro.

4. Se ofrece al grupo un trabajo independiente de los estudiantes sobre el desarrollo de fragmentos específicos de una lección.

5. Se realiza la corrección y enriquecimiento de una experiencia grupal.

6. Se ofrece un trabajo independiente sobre el desarrollo de fragmentos específicos de la lección con la verificación posterior por parte del profesor de manera individual.

7. Se realiza la corrección y enriquecimiento de una experiencia individual de cada uno.

8. Se forma la experiencia subjetiva colectiva en forma de un conjunto de presentaciones en el sistema electrónico de formación universitaria. También se recomienda que los estudiantes preparen las publicaciones que generalizan su experiencia en el tema.

Presentemos la formulación de tareas para el desarrollo de proyectos metódicos, reflejando los requisitos para los resultados de su creación, e indiquemos el propósito de sus subsecciones.

Tarea para desarrollar un proyecto sobre la metodología para trabajar con una tarea matemática:

1. Determinar el tipo de tareas a resolver (encabezado de presentación) (la determinación del tipo de tareas se dirige a los estudiantes para desarrollar métodos comunes para resolverlas).

2. Distinguir las bases teóricas para resolver las tareas de este tipo y tener en cuenta la actividad de los estudiantes al trabajar con el material teórico (la primera diapositiva) (una de las razones de las dificultades de aprendizaje de los estudiantes está relacionada con su ignorancia de los fundamentos teóricos o métodos de su aplicación en las tareas; por lo tanto, es deseable prever los problemas de los estudiantes y encontrar las formas de su liquidación).

3. Divulgar el diálogo con los estudiantes en cuatro etapas de trabajo con la tarea: análisis de la condición con la compilación simultánea de una entrada corta; buscar una solución que resulte en un plan de decisión; ejecución de decisiones; resumir los resultados del trabajo en la tarea (una diapositiva separada para cada etapa) (una de las razones de las dificultades del maestro para desarrollar la 
metodología para enseñar a los estudiantes a resolver las tareas está asociada con la omisión o mezcla de etapas de trabajo individuales en el problema, entonces la división en diapositivas bajo el principio "la etapa cambia - el principio de diapositivas cambia" es necesariamente).

Tarea para desarrollar un proyecto sobre la metodología de trabajo con un teorema:

1. Para definir el título del teorema (encabezado de presentación) (es importante determinar el lugar de un teorema en el sistema de conocimiento del alumno, por lo tanto, se recomienda dar los nombres a los teoremas).

2. Para divulgar el diálogo con los estudiantes en seis etapas de trabajo con el teorema (una diapositiva separada para cada etapa):

- Motivación del teorema (la etapa de motivación de un teorema particular no se presenta ni en los libros de texto ni en la literatura metódica, por lo tanto, la búsqueda de las formas de motivación es una de las tareas creativas de un estudiante);

- Análisis de la formulación del teorema con construcción simultánea del dibujo;

- Buscar una forma de prueba, que termina con la elaboración de un plan de prueba (¿las preguntas "¿Cómo adivinar que es necesario realizar una construcción adicional, para utilizar un método particular, un teorema particular?" reflexión, que a su vez afecta el pensamiento metódico de los estudiantes);

- Trabajar con el texto de prueba en el libro de texto (es importante aprender a ver a los estudiantes asociados con los textos de prueba en los libros de texto para aprender cómo superar estos problemas);

- Formulación de prueba (es importante aprender a estructurar la prueba, presentando las etapas de prueba y la lógica de cada conclusión);

- Resumen de los resultados del trabajo sobre el teorema (la etapa tiene como objetivo enriquecer la experiencia de los estudiantes con respecto al estudio del teorema, la construcción del problema "en un solo paso", demostrando los conceptos básicos de su uso en situaciones específicas).

Tarea para desarrollar un proyecto sobre la metodología para trabajar con el texto matemático:

1. Definir el problema educativo del texto (encabezado de presentación) (el problema educativo del texto es el núcleo alrededor del cual se construyen las actividades cognitivas y reflexivas de los estudiantes).

2. Para dividir el texto en partes usando la regla: "el objetivo cambia - la etapa cambia" y tener en cuenta la actividad de los estudiantes cuando trabajan dividiendo el texto en partes (la primera diapositiva) (la capacidad de determinar El propósito de cada texto es importante tanto para el alumno como para el profesor).

3. Divulgar el diálogo con los estudiantes en cada parte del texto en torno a preguntas como: "¿Qué trata la parte del texto?", "¿Qué aprendiste de esta parte?" (una diapositiva separada para cada parte del texto). (Las preguntas presentadas ayudan a reelaborar el texto en la dirección de su comprensión). 


\section{ORANGE JOURNAL}

El papel del texto educativo en la educación intelectual de los estudiantes se revela en el documento (Holodnaya y Gelfman, 2016).

La preparación de los comentarios metódicos a cada diapositiva por parte de los estudiantes, en la que se recomienda indicar el propósito de las actividades de los estudiantes y los métodos que aseguran su éxito independiente en el logro de este objetivo, ayuda a aumentar el uso del proyecto metódico como un método para enseñar a los futuros maestros. Esto permite a los desarrolladores de proyectos repensar sus soluciones metódicas. Al mismo tiempo, se utiliza un diálogo con el profesor mediante correo electrónico.

\section{Resultados}

Proyecto colectivo: "Aprendizaje y enseñanza para resolver problemas geométricos", incluido el alumno presentaciones de computadora, se convirtió en el ganador del 1er Concurso Bryansk Network por el mejor material educativo "Solicitud de éxito" en la nominación "El mejor desarrollo metódico de la lección de matemáticas usando las TIC" en el marco del programa Intel "Aprendizaje para el futuro" en Rusia, en 2007.

Los proyectos metódicos de los estudiantes de los años siguientes se presentan en el sistema electrónico de formación universitaria.

Varios estudiantes resumieron los resultados de su investigación en el marco de conferencias sobre problemas contemporáneos de la enseñanza de las matemáticas en Rusia y Ucrania.

Los proyectos se probaron en el marco de la VIII Escuela Republicana de Matemáticas de Verano para Maestros (Vladikavkaz) (Abaturova y Malova, 2017).

Las conclusiones que reflejan la experiencia colectiva y relacionadas con proyectos para trabajar con el texto, problemas planimétricos o estereométricos, en el estudio de teoremas se presentan en el documento (Smirnova y Yaroslavl, 2010).

\section{Discusión}

Definamos una serie de problemas que requieren más investigación.

- Problema de tiempo. El desarrollo de un proyecto metódico requiere esfuerzos considerables que requieren mucho tiempo tanto del alumno como del profesor para su análisis.

- El problema del uso del proyecto en la práctica docente. Se probaron las siguientes opciones: un estudiante usa su proyecto o proyecto de otro estudiante en la práctica de enseñar a estudiantes; los maestros utilizan los proyectos durante su participación en el juego metódico; Los proyectos se recomiendan para el uso independiente. El análisis de estas y otras posibles opciones requiere una investigación.

- Problema de diálogo. El diálogo educativo asume tanto la variabilidad de las respuestas de los estudiantes como la duración de las pausas entre la pregunta y la 
respuesta, que no siempre es posible estipular en una presentación por computadora.

\section{Resumen}

Los proyectos metódicos deberían dedicarse a aquellas cuestiones de matemáticas, que causan dificultades a los estudiantes (problemas complejos, pruebas de teoremas, análisis semántico de texto matemático, etc.)

El nivel de los proyectos metódicos debe corresponder a los requisitos modernos del enfoque de la actividad, la capacitación orientada personalmente, el uso de las TIC. La inclusión de los estudiantes en la creación de proyectos metódicos es el medio efectivo para capacitar a los maestros.

\section{Conclusiones}

El trabajo experimental a largo plazo muestra que los proyectos metódicos resuelven tres problemas: 1) motivar el desarrollo independiente de un producto metódico; 2) proporcionar asistencia matemática efectiva a los estudiantes, ya que contribuyen a garantizar su éxito independiente, así como asistencia metódica al alumno (maestro) en el desarrollo de una solución metódica moderna; 3) enriquecer la metodología de enseñanza de las matemáticas a los nuevos desarrollos.

\section{Expresiones de gratitud}

El trabajo se realiza de acuerdo con el Programa de Crecimiento Competitivo del Gobierno Ruso de la Universidad Federal de Kazan.

\section{Referencias}

Abaturova V.S., Malova I.E. (2017) Condiciones para garantizar la efectividad de un juego metódico profesional como forma de organización de la formación de docentes. Tecnologías educativas y sociedad, vol. 20, núm. 3, págs. 257-269.

Fisher T., Higgins C., Loveless A. (2006). Docentes que aprenden con tecnologías digitales: una revisión de investigaciones y proyectos. Bristol: Futurelab, (https://www.nfer.ac.uk/publications/FUTL67). Goos M. (2004). Aprendiendo Matemáticas en una Comunidad de Investigación en el Aula. Revista de Investigación en Educación Matemática, vol. 35, núm. 4, págs. 258-291.

Holmqvist M. (2011). El aprendizaje de los docentes en un estudio de aprendizaje, Ciencias de la instrucción. Vol. 39, núm. 4, págs. 497-511.

Holodnaya M.A., Gelfman E.G. (2016) El desarrollo de textos educativos como el medio de

Educación intelectual de los estudiantes. M.: Editorial "Instituto de Psicología de RAS", p. 200. Koehler M.J., Mishra P. (2005), Teachers Learning Technology by Design, Journal of Computing in Teacher Education. Vol. 21, núm. 3, págs. 94-102.

Malova I.E. (2017) Proyectos metódicos de los alumnos como medio para mejorar la calidad de la formación del futuro docente. N.I. Lobachevsky y la educación matemática 


\section{ORANGE JOURNAL}

en Rusia: Materiales del Foro internacional sobre educación matemática, 18-22 de octubre de 2017 / Ed. por L.I. Shakirova Kazan: Editorial de la Universidad de Kazan, vol. 1, págs. 238-242.

Mason J., Spence M. (1999). Más allá del mero conocimiento de las matemáticas: la importancia de saber actuar en el momento. Formas de conocimiento matemático: aprendizaje y enseñanza con entendimiento Educational Studies in Mathematics, pp. 135161, (https://link.springer.com/book/10.1007/978-

94-017-1584-3).

Smirnova E.I., Yaroslavl. (2010) Modelado visual en la enseñanza de las matemáticas: teoría y práctica: libro de texto / Ed.

Tchoshanov, M., Quinones, M.C., Shakirova, K.B., Ibragimova, E.N., Shakirova, L.R. (2017) Analizando las conexiones entre el conocimiento específico del tema del profesor y el alumno de matemáticas de secundaria inferior. Journal of Mathematical Behavior, 47, págs. 54-69...

Yakimanskaya I.S. (2014) Fundamentos de la educación orientada personalmente. M.: BINOM. Laboratoriya Znaniy, pág. 220 